\title{
Newly discovered active binaries in the RasTyc sample of stellar X-ray sources ${ }^{\star}, \star \star$
}

\section{Orbital and physical parameters of six new binaries}

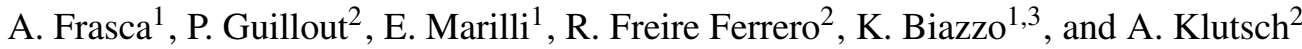 \\ 1 INAF - Osservatorio Astrofisico di Catania, via S. Sofia, 78, 95123 Catania, Italy \\ e-mail: afrasca@oact.inaf.it \\ 2 Observatoire Astronomique, UMR 7550, 11 rue de l'Université, 67000 Strasbourg, France \\ 3 Dipartimento di Fisica e Astronomia, Università di Catania, via S. Sofia, 78, 95123 Catania, Italy
}

Received 23 November 2005 / Accepted 17 March 2006

\section{ABSTRACT}

\begin{abstract}
We present the first results from follow-up optical observations, both photometric and spectroscopic, of stellar X-ray sources, selected from the RasTyc sample, resulting from the cross-correlation of ROSAT All-Sky Survey (RASS) and TYCHO catalogues. In particular, we report on the discovery of six late-type binaries, for which we obtained good radial velocity curves and solved their orbits. We performed an automatic spectral classification of both single-lined and double-lined binaries with codes developed by us and found two binaries composed of two main-sequence stars and four binaries with an evolved (giant or subgiant) component. Filled-in or pure emission $\mathrm{H} \alpha$ profiles indicative of a moderate or high level of chromospheric activity were observed. In nearly all the systems, we also detected a photometric modulation ascribable to surface inhomogeneities that is correlated with the orbital period, suggesting a synchronization between rotational and orbital periods. The position on the HR diagram of the components of the five sources with a known parallax indicates three binaries containing only main-sequence stars and two single-lined systems with a giant component. The kinematical properties of two, or possibly four, of the observed systems are consistent with a young disk population.
\end{abstract}

Key words. stars: binaries: spectroscopic - stars: fundamental parameters - stars: late-type - stars: activity - stars: kinematics X-rays: stars

\section{Introduction}

The X-ray emission of late-type stars $(\mathrm{F}-\mathrm{M})$ is thought to be caused by surface magnetic activity generated by dynamo action in their subphotospheric convective zones. As a star gets older, the efficiency of the dynamo action declines because of the angular momentum loss produced by the stellar wind. Thus, sources with a high X-ray emission level are thought to be the youngest stars in the solar neighborhood.

X-ray sky surveys, performed in recent years, have led to the detection of thousands of active late-type stars in the field and to the discovery of associated young galactic stellar structures often unnoticed at optical wavelengths. A young latetype stellar population associated with the Gould's belt was discovered by Guillout et al. (1998) by means of the crosscorrelation of the largest catalog of X-ray sources, the ROSAT All-Sky Survey ( $\simeq 150000$ sources), with the TYCHO catalog ( $\simeq 1000000$ stars). The so-called RasTyc sample, containing about 14000 stellar X-ray sources, was thus obtained (Guillout et al. 1999). Wichmann et al. (2003) report on a tight kinematic group of ten stars younger than the Pleiades (100 Myr) among the 748 optical counterparts of X-ray sources investigated. They also found $43 \%$ of the sample with detectable lithium, indicating

^ Based on observations collected at the OHP (France) and Catania Astrophysical Observatory (Italy)

$\star \star$ Tables 2 to 7 are only available in electronic form at the CDS via anonymous ftp to cdsarc.u-strasbg.fr $(130.79 .128 .5)$ or via http://cdsweb.u-strasbg.fr/cgi-bin/qcat?]/A+A/454/301 fairly young stars. Zickgraf et al. (2005) performed an analogous study of another sample of $\sim 200$ late-type stellar X-ray sources, finding half of the G-K stars to be younger than the Hyades ( $600 \mathrm{Myr})$ and about $25 \%$ of the sample comparable in age to the Pleiades, with a small subsample of ten very young (PMS) stars.

The dependence on age of stellar activity offers a unique opportunity to investigate the local star-formation history and stellar birthrate, as well as the chemical evolution of stars over the past few billion years. Moreover, the formal optical identification of a large number of X-ray sources and the subsequent determination of their physical and kinematical parameters is a necessary task first in addressing fundamental questions about convection and other internal mixing processes, angular momentum evolution of late-type stars, and distribution of surface activity on rapidly rotating young stars and ultimately in putting constraints on dynamo mechanisms.

Thus, we started a spectroscopic observation campaign aimed at a deep characterization (nature and evolutionary status) of a representative sub-sample of the RasTyc population. About 1200 late-type (F-M spectral-type range) X-ray bright (ROSAT PSPC count-rate $>0.03 \mathrm{cts} / \mathrm{s}$ ) field stars scattered across the northern sky were initially selected. Candidates already with good data from the literature were then discarded, while the remaining stars were chosen for high-resolution spectroscopic follow-up observations. A preliminary program (110 targets) was activated with the ELODIE echelle spectrograph at the $193-\mathrm{cm}$ telescope of the Observatoire de Haute 
Table 1. Main parameters of the RasTyc sources.

\begin{tabular}{lcccccccc}
\hline \hline Name & $\begin{array}{c}\alpha(2000) \\
\mathrm{h} \mathrm{m} \mathrm{s}\end{array}$ & $\begin{array}{c}\delta(2000) \\
{ }^{\prime}{ }^{\prime \prime}\end{array}$ & $\begin{array}{c}\pi \\
(\mathrm{mas})\end{array}$ & $\begin{array}{c}\mu_{\alpha} \\
(\mathrm{mas} / \mathrm{yr})\end{array}$ & $\begin{array}{c}\mu_{\delta} \\
(\mathrm{mas} / \mathrm{yr})\end{array}$ & $\begin{array}{c}\text { Ref. }^{a} \\
\text { X-ray source } \\
\text { 1RXS }\end{array}$ & $\begin{array}{c}\text { Counts } \\
(\mathrm{ct} / \mathrm{s})\end{array}$ \\
\hline HD 183957 & 193137.0 & +114326 & $24.9 \pm 8.9$ & 76.7 & -22.2 & $\mathrm{~T}$ & $\mathrm{~J} 193135.6+114331$ & $3.02 \times 10^{-2}$ \\
BD+15 4538 & 215940.1 & +160218 & $14.53 \pm 1.63$ & -75.6 & -66.8 & $\mathrm{H}$ & $\mathrm{J} 215940.8+160220$ & $3.31 \times 10^{-1}$ \\
BD+33 4462 & 221428.3 & +335630 & - & 45.0 & 12.8 & $\mathrm{~T}$ & $\mathrm{~J} 221428.3+335634$ & $1.92 \times 10^{-1}$ \\
HD 237215 & 040542.3 & +570737 & $2.14 \pm 1.29$ & -16.4 & -5.7 & $\mathrm{H}$ & $\mathrm{J} 040542.8+570733$ & $2.77 \times 10^{-1}$ \\
HD 57267 & 072132.8 & +260933 & $4.31 \pm 1.25$ & -2.9 & -10.9 & $\mathrm{H}$ & $\mathrm{J} 072132.9+260939$ & $2.80 \times 10^{-1}$ \\
BD+38 2140 & 102622.7 & +374513 & $27.9 \pm 18.7$ & -89.9 & 21.9 & $\mathrm{~T}$ & $\mathrm{~J} 102622.8+374515$ & $1.84 \times 10^{-1}$ \\
\hline
\end{tabular}

${ }^{a} \mathrm{H}=$ HIPPARCOS parallax and proper motions; $\mathrm{T}=\mathrm{TYCHO}-1$ parallax and TYCHO-2 proper motions.

Provence (OHP). The remaining stars that were brighter than $V=10$. 0 were subsequently scheduled as a key-program on the OHP 152-cm telescope with the AURELIE spectrograph. Each target was planned to be observed in the $\mathrm{H} \alpha$ (chromospheric activity diagnostic) and lithium (age diagnostic) spectral domains.

As a matter of fact, high-resolution spectroscopic observations allow us to derive spectral types and luminosity classes, to infer ages, and to single out SB1 (variable radial velocity) and SB2 spectroscopic binaries. Rotational velocities (from Doppler broadening) and chromospheric activity levels (from $\mathrm{H} \alpha$ emission) can also be determined. More than 600 stars have been observed so far. A paper by Guillout et al. dealing with the global properties of the stars investigated with this spectroscopic survey is in preparation.

The preliminary analysis of the ELODIE and AURELIE spectra reveals that spectroscopic binaries represent a large fraction of our RasTyc subsample. Crude statistics show that both SB1 and SB2 systems could account for more than $50 \%$ of the sample. In soft X-ray surveys, neither the fraction of BY Dra binaries nor the contamination by older RS CVn systems (for which the high magnetic activity level results from the synchronization of rotational and orbital periods) are actually known. The knowledge of the incidence of binaries in this sample is of paramount importance for studying the recent local star formation history. Moreover, we are especially interested in the SB2 systems with large spectral-line separation, which are mainly close binaries. The short-period binary frequency is likely affected by different environments and initial conditions during the evolution from the pre-main sequence (PMS) to the main sequence (MS) phase (Brandner \& Koehler 1998; Duchêne 1999). However, more statistics of orbital parameters are needed in order to shed light on the remaining problems with the orbital and rotational evolution of close binaries $(P<10 \mathrm{~d})$, like the tidal synchronization and circularization processes. For this reason, we are monitoring more than 30 of these new binaries, both photometrically and spectroscopically, with the $91-\mathrm{cm}$ telescope of the Osservatorio Astrofisico di Catania (OAC).

In this paper we present a study of three single-lined (SB1) and three double-lined (SB2) late-type binaries for which we have complete data sets. A detailed analysis of the entire sample of stellar X-ray sources and of the close binaries discovered is absolutely necessary for drawing statistically significant conclusions. As such, the six objects presented in this paper cannot be considered as representative of the close binary population of soft X-ray surveys.

For these binaries we obtained good radial velocity curves and orbital solutions (Sect. 3.1). The physical parameters (spectral types, $v \sin i$ 's, lithium abundances, etc.), the photospheric and chromospheric activity, and the kinematics of these sources are discussed in Sect. 3. The coronal activity level is given by the $\mathrm{X}$-ray luminosity, computed from the ROSAT PSPC count rate, and is discussed in Sect. 3 as well. The conclusions are presented in Sect. 4.

\section{Observations and data reduction}

The six binaries studied in this work are listed in Table 1 together with relevant information from the literature. Three sources are also included in the HIPPARCOS catalog (ESA 1997) and have good parallaxes $(\pi)$ and proper motions $\left(\mu_{\alpha}, \mu_{\delta}\right)$. For the other stars we used the proper motions given in the TYCHO-2 catalog (Hog et al. 2000) that have a typical precision of 2.5 mas $\mathrm{yr}^{-1}$. Both HD 183957 and BD+38 2140 are nearby stars with reliable TYCHO parallaxes (35\% and 65\% errors, respectively). The parallax of BD+334462 is not known, since there is only an entry of -6.6 mas in the TYCHO-1 catalog (ESA 1997) for this star.

\subsection{Spectroscopy}

Spectroscopic observations were obtained at the OHP and at the M.G. Fracastoro station (Serra La Nave, Mt. Etna, $1750 \mathrm{~m}$ a.s.1.) of the OAC.

At the OHP in 2000 and 2001 we observed with the ELODIE echelle spectrograph connected to the $193-\mathrm{cm}$ telescope. The 67 orders recorded by the CCD detector cover the 3906-6818 $\AA$ wavelength range with a resolving power of about 42000 (Baranne et al. 1996). Typically, we acquired a couple of spectra in different nights for each source, with the exception of HD 183957 and BD+33 4462, for which we have 12 and 11 ELODIE spectra, respectively. The signal-to-noise ratio $(S / N)$ of these spectra was typically between 60 and 90 , though we estimated it as low as 30 for the faintest stars in bad sky conditions. The ELODIE spectra were automatically reduced on-line during the observations, and the cross-correlation with a reference mask was produced as well.

For HD 237215 we have no ELODIE spectrum, but we observed this source in 2001 at the OHP with the AURELIE spectrograph of the coude T152 telescope in the $\mathrm{H} \alpha$ and Li I spectral regions and obtained a total of ten spectra. We used grating N.7, which yields a spectral resolution of about 40000 at the wavelengths of our observations. The $\mathrm{S} / \mathrm{N}$ of these spectra was about 80-100. All spectra obtained on the OHP T152 telescope were corrected for bias and flat field using standard MIDAS procedures. After cosmics removal, Th-Ar arc lamp exposures were used to define the wavelength scale of our spectra. Telluric lines in the $\mathrm{H} \alpha$ region were removed following the method presented in Frasca et al. (2000).

The observations carried out at Catania Observatory have been performed in 2001, 2002 and 2003 with the echelle spectrograph FRESCO at the $91-\mathrm{cm}$ telescope. The spectrograph is fed by the telescope through an optical fiber (UV - NIR, $200 \mu \mathrm{m}$ 
core diameter) and is placed in a stable position in the room below the dome level. Spectra were recorded on a CCD camera equipped with a thinned back-illuminated SITe CCD of $1024 \times 1024$ pixels $($ size $24 \times 24 \mu \mathrm{m}$ ). The echelle configuration with the 900 -line/mm cross-disperser yields a resolution of about 14000 , as deduced from the FWHM of the lines of the Th-Ar calibration lamp. The observations were made in the red region. The detector allowed us to record five orders in each frame, spanning from about 5860 to $6700 \AA$. We obtained from 13 to 26 OAC spectra for each source with an $\mathrm{S} / \mathrm{N}$ ratio ranging from 20 to 200 , depending on the star magnitude and sky conditions. The OAC data reduction was performed by using the ECHELLE task of IRAF $^{1}$ package following the standard steps: background subtraction, division by a flat-field spectrum given by a halogen lamp, wavelength calibration using the emission lines of a Th-Ar lamp, and normalization to the continuum through a polynomial fit.

\subsection{Photometry}

The photometric observations were carried out in 2001 and 2002 in the standard $U B V$ system with the 91-cm telescope of $\mathrm{OAC}$ and a photon-counting refrigerated photometer equipped with an EMI 9789QA photomultiplier, cooled to $-15^{\circ} \mathrm{C}$. The dark noise of the detector, operated at this temperature, is about 1 photon/s.

For each field of the RasTyc sources, we chose two or three stars with known $U B V$ magnitudes to be used as local standards for determining the photometric instrumental "zero points". Additionally, several standard stars, selected from the list of Landolt (1992), were also observed during the run in order to determine the transformation coefficients to the Johnson standard system.

A typical observation consisted of several integration cycles (from 1 to 3 , depending on the star brightness) of 10, 5, $5 \mathrm{~s}$, in the $U, B$, and $V$ filters, respectively. A $21^{\prime \prime}$ diaphragm was used. The data were reduced by means of the photometric data reduction package PHOT designed for the photoelectric photometry of Catania Observatory (Lo Presti \& Marilli 1993). Seasonal mean extinction coefficients for Serra La Nave Observatory were adopted for the atmospheric extinction correction.

The photometric errors, estimated from measurements of standard stars with a brightness comparable to the program stars, are typically $\sigma_{V}=0.005$ and $\sigma_{B-V}=0.007$.

\section{Results}

\subsection{Radial velocity}

The radial velocity (RV) measurements for the ELODIE data were performed thanks to the cross-correlation functions (CCFs) produced on-line during the data acquisition. The RV uncertainty due to the photon noise was calculated according to Eq. (9) of Baranne et al. (1996) and always resulted in a few tens $\mathrm{m} \mathrm{s}^{-1}$. However, since the ELODIE spectra were acquired in the standard mode (object+sky) without a simultaneous recording of ThAr spectrum in the second optical fiber, we adopted the typical velocity variation of $150 \mathrm{~m} \mathrm{~s}^{-1}$ due to the overall stability of the spectrograph (Baranne et al. 1996) as measure error.

${ }^{1}$ IRAF is distributed by the National Optical Astronomy Observatories, which are operated by the Association of Universities for Research in Astronomy, Inc., under cooperative agreement with the National Science Foundation.
The RV measurements for OAC and AURELIE spectra were obtained by means of the cross-correlation of each echelle order of the targets with the corresponding one of a bright RV standard star (e.g., Tonry \& Davis 1979; Fitzpatrick 1993). For this purpose the IRAF task FXCOR was used. We excluded the $\mathrm{H} \alpha$ and $\mathrm{NaI} \mathrm{D}_{2}$ lines from the analysis, because they can be contaminated by chromospheric emission. All the spectral ranges heavily affected by telluric absorption lines (e.g., the $\mathrm{O}_{2}$ band 16276-6315) were also discarded. The radial velocity measurements of these new binaries, listed in Tables 2-7 together with their standard errors $(\Delta V)$, were obtained by averaging RV data from all echelle orders with the usual instrumental weight $W_{\mathrm{i}}=$ $\sigma_{\mathrm{i}}^{-2}$. The $\sigma_{\mathrm{i}}$ values were computed by FXCOR according to the fitted peak height and the antisymmetric noise as described by Tonry \& Davis (1979). The standard errors of the weighted means were computed on the basis of the errors $\sigma_{\mathrm{i}}$ in the RV values for each order according to the usual formula (see, e.g., Topping 1972).

To better evaluate the centroids of the CCF peaks for each echelle order (i.e. the radial velocity of the primary and secondary components), we adopted two separate Gaussian fits for the cases with a significant peak separation (i.e. far from the conjunctions), and a two-Gaussian fit algorithm to resolve cross-correlation peaks in the cases of partial blending of the spectral lines. At orbital phases very close to the conjunctions, only one peak could be seen in the CCF and consequently only a "blended" RV could be measured.

The observed RV curves are displayed in Fig. 1, where for SB2 systems we used filled symbols for the primary (more massive) components and open symbols for the secondary ones. The RVs obtained in fully blended situations are marked with crosses and were not used for the RV solutions. We initially searched for eccentric orbits and, in any case, found very low eccentricity values (e.g., $e=0.01$ for HD 183957, $e=0.03$ for $\mathrm{BD}+334462$ ). Thus, following the precepts of Lucy \& Sweeney (1971), we adopted $e=0$. The CURVEFIT routine (Bevington 1969) was used to fit the observed RV curves. This algorithm also provides us with the errors on the fitting parameters. The circular solutions are also represented in Fig. 1. It is worth noticing that all systems, with the exception of $\mathrm{BD}+154538$, have larger periods than the cut-off value of 7.56 days found by Melo et al. (2001) for orbital circularization in PMS binaries. This excludes PMS among at least five of our six binaries.

The orbital period $\left(P_{\text {orb }}\right)$, barycentric velocity $(\gamma)$, RV semiamplitudes $(k)$, and masses $\left(M \sin ^{3} i\right)$ for each binary system are listed in Table 8 , where $\mathrm{P}$ and $\mathrm{S}$ refer to the primary and secondary components of the SB2 systems, respectively.

\subsection{Photometry}

With the exception of HD 183957, for which no modulation is visible whether in OAC data or in TYCHO $V_{\mathrm{T}}$ magnitudes, and of $\mathrm{BD}+154538$, which displays small photometric variations, all sources show a photometric modulation that is well correlated with the orbital period. This high degree of synchronization between orbit and rotation in all systems with detected rotational modulation, together with the very low (or null) orbit eccentricity and the low lithium abundance (see Sect. 3.6), is indicative of binary systems in evolutionary stages later than the zero-age main-sequence (ZAMS). In fact, according to the prediction of Zahn \& Bouchet (1989), the rotation period at this stage should be significantly shorter (roughly one half) than the orbital one and the synchronization is attained at ages of a few hundred Myr. 

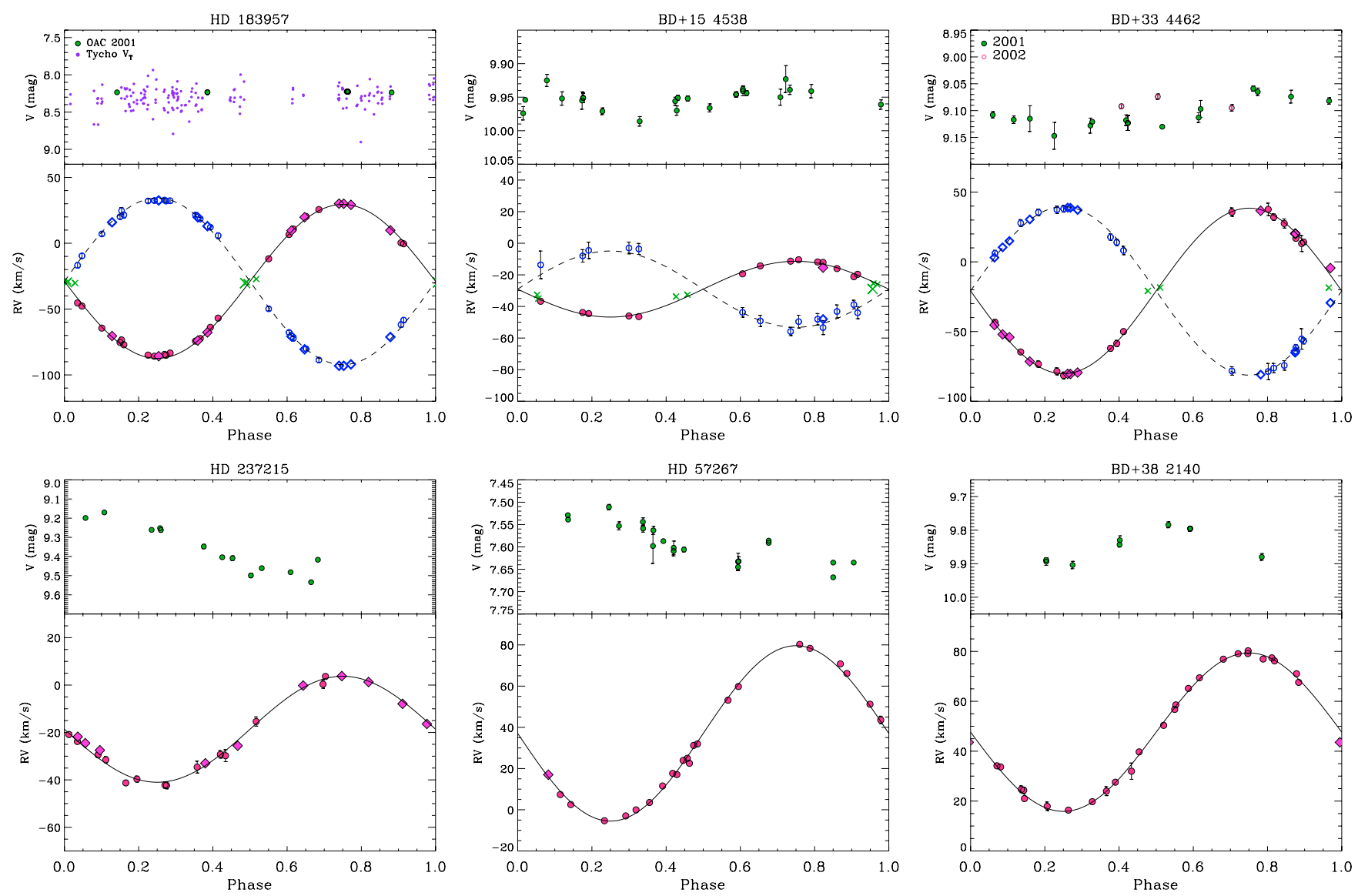

Fig. 1. Radial velocity curves (circles $=$ OAC data, diamonds $=$ OHP data) of the new six RasTyc binaries. For the three SB2 systems, filled and open symbols for the primary (more massive) and secondary components have been used, respectively. The solid and dashed lines represents the circular solutions for the primary and secondary components, respectively. The contemporaneous $V$ photometry is displayed, as a function of the orbital phase, on the top panel of each box.

Table 8. Orbital parameters of the new binaries.

\begin{tabular}{lcccccc}
\hline \hline Name & $\begin{array}{c}\mathrm{HJD0}^{a} \\
(2450000+)\end{array}$ & $\begin{array}{c}P_{\text {orb }} \\
\text { (days) }\end{array}$ & $\begin{array}{c}\gamma \\
\left(\mathrm{km} \mathrm{s}^{-1}\right)\end{array}$ & $\begin{array}{c}k \\
\left(\mathrm{~km} \mathrm{~s}^{-1}\right) \\
{[\mathrm{P} / \mathrm{S}]}\end{array}$ & $\begin{array}{c}M \sin ^{3} i \\
\left(M_{\odot}\right) \\
{[\mathrm{P} / \mathrm{S}]}\end{array}$ & $M_{1} / M_{2}$ \\
\hline HD 183957 & 1759.550 & 7.954 & $-29.0(2)$ & $58.47(6) / 63.29(6)$ & $0.773(2) / 0.714(2)$ & $1.082(2)$ \\
BD+15 4538 & 1642.767 & 1.756 & $-29.1(3)$ & $17.3(3) / 24.1(1.3)$ & $0.0077(9) / 0.0056(4)$ & $1.37(4)$ \\
BD+33 4462 & 1208.480 & 10.121 & $-20.9(1)$ & $59.38(6) / 60.41(6)$ & $0.909(2) / 0.894(2)$ & $1.017(2)$ \\
HD 237215 & 2209.52 & 12.63 & $-18.6(2)$ & $22.4(4)$ & SB1 & \\
HD 57267 & 2196.58 & 36.24 & $+37.1(2)$ & $42.6(2)$ & SB1 & \\
BD+38 2140 & 2207.117 & 15.465 & $+47.6(1)$ & $31.8(2)$ & SB1 & \\
\hline
\end{tabular}

${ }^{a}$ Heliocentric Julian date of the inferior conjunction of the primary (more massive) component.

For BD +154538 , the low amplitude of its photometric variations and the very low values of $M \sin ^{3} i$ imply a very low inclination of the orbital/rotational axis.

\subsection{Spectral type and $v \sin i$ determination}

The projected rotational velocity, $v \sin i$, was measured by means of the ELODIE CCFs and the calibration relation between CCF width and $v \sin i$ proposed by Queloz et al. (1998) and is reported in Table 9. The lowest rotation rate $\left(v \sin i \simeq 4 \mathrm{~km} \mathrm{~s}^{-1}\right)$ has been detected for both components of HD 183957, which also display the lowest $\mathrm{H} \alpha$ activity level compared to the other sources (see Sect. 3.5). From the $v \sin i$ and the rotational period, assumed to be equal to the orbital period, we have calculated, for each star, the minimum radius $R \sin i$. The values of $R \sin i$ are listed in Table 9.

For the SB1 systems observed with ELODIE, we determined effective temperatures and gravity (i.e. spectral classification) by means of the TGMET code, available at the OHP (Katz et al. 1998). We also used ROTFIT, a code written by one of us (Frasca et al. 2003) in IDL (Interactive Data Language, RSI) to simultaneously find the spectral type and the $v \sin i$ of the target searching, into a library of standard star spectra, the spectrum that gives the best match (minimum of the residuals) to the target one, after the rotational broadening. As a standard star library, we used a sub-sample of the TGMET list composed of 87 stars that is well-distributed in effective temperature and gravity and in a suitable range of metalicities. The standard star spectra 
Table 9. Physical parameters of the new binaries.

\begin{tabular}{|c|c|c|c|c|c|c|c|}
\hline Name & $B-V$ & $\begin{array}{c}v \sin i \\
\left(\mathrm{~km} \mathrm{~s}^{-1}\right) \\
{[\mathrm{P} / \mathrm{S}]}\end{array}$ & $\begin{array}{c}\text { Sp. Type } \\
{[\mathrm{P} / \mathrm{S}]}\end{array}$ & $w_{5500}^{\mathrm{P}}$ & $\begin{array}{c}T_{\text {eff }} \\
(\mathrm{K}) \\
{[\mathrm{P} / \mathrm{S}]}\end{array}$ & $\begin{array}{l}W_{\mathrm{LiI}} \\
(\mathrm{m} \AA) \\
{[\mathrm{P} / \mathrm{S}]}\end{array}$ & $\begin{array}{c}R \sin i^{a} \\
R_{\odot} \\
{[\mathrm{P} / \mathrm{S}]}\end{array}$ \\
\hline HD 183957 & 0.84 & $4.0 / 4.4$ & K0V/K1V & $0.584 \pm 0.006$ & $5150 / 5050$ & $<10$ & $0.63 / 0.69$ \\
\hline$B D+154538$ & 0.79 & $9.2 / 9.4$ & G8-K0V/K3-4V & $0.845 \pm 0.021$ & $5300 / 4600$ & $60 /-$ & $0.32 / 0.32$ \\
\hline$B D+334462$ & 0.70 & $26.5 / 9.7$ & K0III-IV/F7IV & $0.366 \pm 0.016$ & $4800 / 6150$ & $15: /-$ & $5.30 / 1.94$ \\
\hline HD 237215 & 1.14 & 35 & K2III & - & 4350 & - & 8.8 \\
\hline HD 57267 & 0.83 & 40 & G8III & - & 4850 & 70 & 28.6 \\
\hline $\mathrm{BD}+382140$ & 1.03 & 11.5 & K1IV & - & 4750 & 40 & 3.5 \\
\hline
\end{tabular}

${ }^{a}$ Evaluated assuming synchronism between orbital and rotational motions.

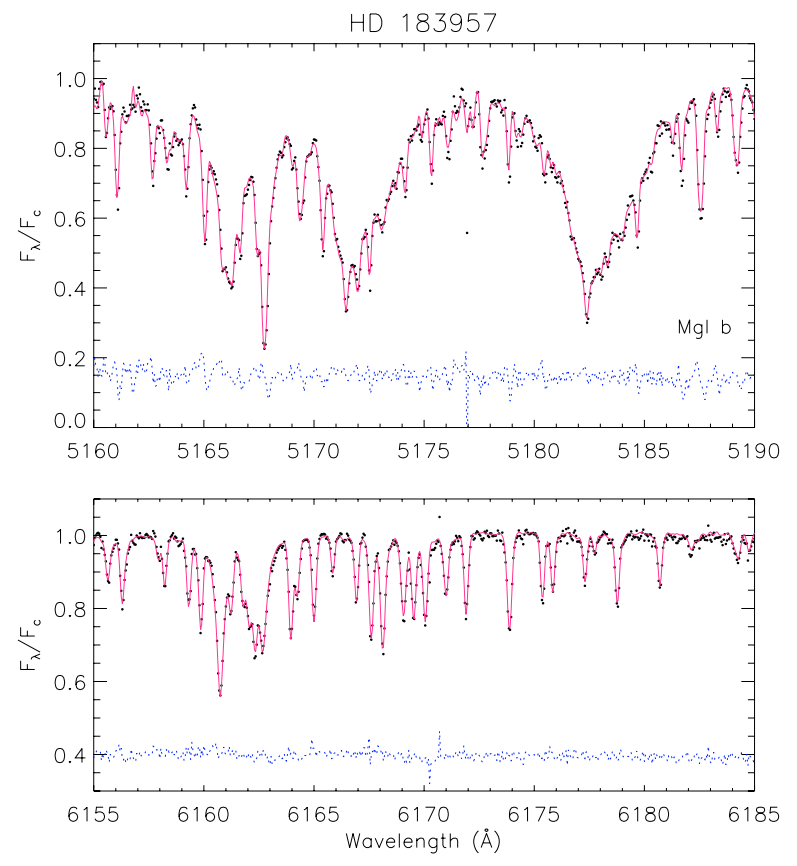

Fig. 2. Observed ELODIE spectrum of the SB2 binary HD 183957 (dots) in the MgIb (upper panel) and the $\lambda 6170 \AA$ (lower panel) spectral regions together with the "synthetic" spectrum over-plotted with a thin line. The latter was built up as the weighted sum of two standard star spectra (K0V and $\mathrm{K} 1 \mathrm{~V}$ for the primary and secondary components, respectively) broadened at the $v \sin i$ of the respective component and Doppler-shifted according to the orbital solution and the orbital phase of the observation $(\phi=0.36)$. The fractional flux contribution of the primary component is 0.62 and 0.58 at 5170 and $6170 \AA$, respectively. Each box also displays the difference (observed - synthetic) with a thin dotted line.

were retrieved from the ELODIE Archive (Prugniel \& Soubiran 2001). The $v \sin i$ values obtained with ROTFIT are in excellent agreement with the ones derived from the CCF width. The ROTFIT code was also applied to the OAC data, using standard star spectra acquired at OAC with the same instrumental setup as for the target spectra. This was especially advantageous for HD 237215, for which we had no ELODIE spectrum.

We also performed an accurate spectral classification for the components of the SB2 systems, thanks to another IDL code made by us, COMPO2, which searches for the best combination of two standard-star spectra able to reproduce the observed spectrum of the SB2 system. Giving the radial velocity and $v \sin i$ of the two components as input parameters, the code finds, for the selected spectral region, the spectral types and fractional flux contributions that reproduce the observed spectrum better,

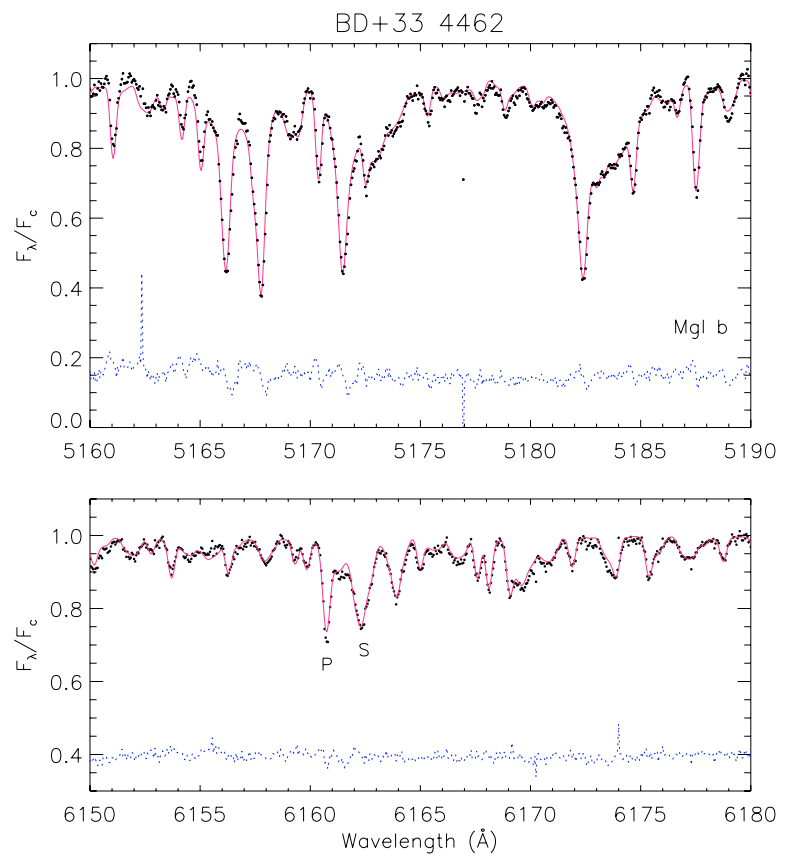

Fig. 3. Observed ElodIE spectrum of BD+334462 (dots) in the MgIb (upper panel) and in the $\lambda 6170 \AA$ (lower panel) regions. The "synthetic" spectrum (F7 IV + K0 III-IV) is displayed with thin lines in the same boxes. Each box also displays the difference (observed - synthetic) with a thin dotted line.

i.e. which minimize the residuals in the collection of difference (observed - synthetic) spectra.

The results of the application of ROTFIT and COMPO2 to different spectral regions, from blue to red wavelengths, are in good agreement (Figs. 2, 3). In addition, the relative contributions of the components of SB2 systems in a given spectral region show a very small dispersion for the best spectral combinations. We list in Table 9 the spectral types, the corresponding effective temperatures, and the relative contributions of the primary components to the spectral continuum at $5500 \AA$, $w_{5500}^{\mathrm{P}}$. The last were obtained by averaging 20 values taken from the five best spectral combinations in four échelle orders around $5500 \AA$.

We find two binaries composed of MS stars, HD 183957 and $\mathrm{BD}+154538$, while the remaining systems contain an evolved star (giant or sub-giant) and can be classified as new RS CVn systems. The spectral classification of the SB1 system $\mathrm{BD}+382140$, as well as the value of $R \sin i \simeq 3.5 R_{\odot}$, indicate a slightly evolved star, but its position in the HR diagram (Sect. 3.4) appears more consistent with a main-sequence star. 


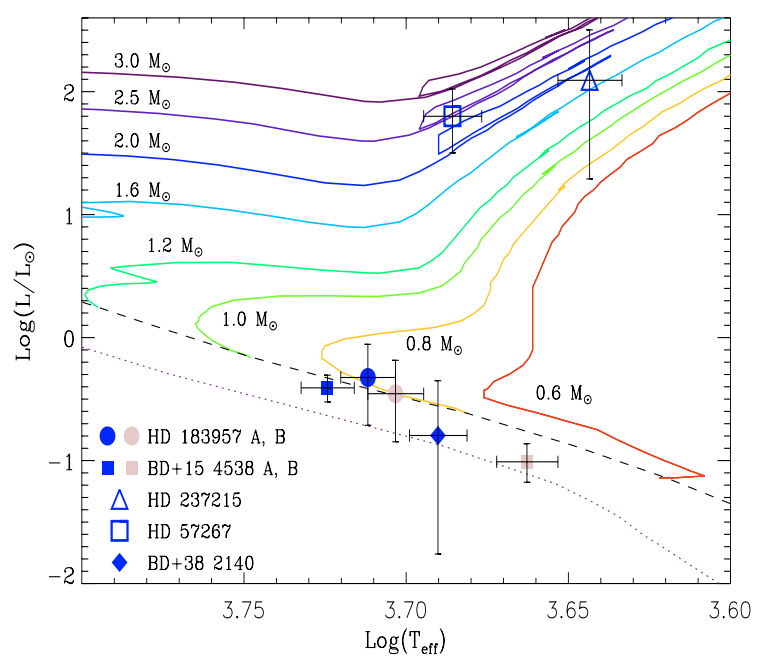

Fig. 4. The HR diagram of the components of the five RasTyc stars with known parallax. The evolutionary tracks for different masses from Girardi et al. (2000) are also shown by continuous lines. The isochrones at $\log$ age $=7.8($ ZAMS $)$ with solar metalicity $(Z=0.019)$ and with $Z=$ 0.001 are also displayed with a dashed and a dotted line, respectively.

\subsection{Position on the HR diagram}

The values of $M_{1,2} \sin ^{3} i$ determined for the SB2 systems from the radial velocity solutions provide lower limits for the masses of binary components. Since all the RasTyc binaries, with the exception of $\mathrm{BD}+334462$, have a reliable parallax from TYCHO or HIPPARCOS, we can place these stars onto the HertzsprungRussel (HR) diagram to check whether the dynamically determined masses are consistent with those inferred from the evolutionary tracks. For this purpose we used the evolutionary tracks and isochrones for low- and intermediate-mass stars calculated by Girardi et al. (2000).

The temperatures were deduced from the spectral classification (Sect. 3.3). We estimated an error on the effective temperature of about 100-150 K, based on the spectral type uncertainty of one spectral subclass. We evaluated the interstellar extinction $A_{V}$ from the system distance, assuming a mean extinction of $1.7 \mathrm{mag} / \mathrm{kpc}$ on the galactic plane $\left(|b|<5^{\circ}\right)$ and $0.7 \mathrm{mag} / \mathrm{kpc}$ out of the plane. The reddening was estimated according to the standard law $A_{V}=3.1 E_{\mathrm{B}-\mathrm{v}}$. However, since nearly all the systems are close to the Sun, the extinction is very low $\left(A_{V} \leq 0^{\mathrm{m}} \cdot 16\right)$. The only exception is HD $237215\left(A_{V}=0.79\right)$, which is close to the galactic plane and is about $450 \mathrm{pc}$ away. For the single-lined binaries, the de-reddened $V_{0}$ magnitude at maximum brightness (minimum spottedness) was converted into absolute magnitude $M_{V}$ with the parallax and subsequently converted into bolometric magnitude by using the bolometric correction tabulated by Flower (1996) as a function of the effective temperature. The bolometric magnitude of the Sun, $M_{\mathrm{bol}}=4.64$ (Cox 2000), was used to express the stellar luminosity in solar units. For SB2 systems we used the luminosity ratio at $5500 \AA$ A deduced by means of the spectral synthesis code (COMPO2) and listed in Table 9 to obtain the $V$ magnitudes of the primary and secondary components from the combined magnitude observed by us. The uncertainty of the stellar luminosity accounts for the parallax and the luminosity-ratio (for SB2 systems) errors.

The HR diagram for the five RasTyc sources with known distance, represented with different symbols, is shown in Fig. 4 where the evolutionary tracks of Girardi et al. (2000) are displayed with continuous lines.
Notwithstanding the relatively large errors, we can confirm the giant evolutionary phase for HD 237215 and HD 57267. From the evolutionary tracks, the latter star should have a mass of $2.5 \pm 0.5 M_{\odot}$, while for the former we can deduce a less secure mass of $1.7 \pm 1.0 M_{\odot}$. Both components of HD 183957 and $\mathrm{BD}+154538$ are main-sequence stars. The masses deduced for HD $183957 \mathrm{~A}$ and B from their position along the main sequence are about 0.88 and $0.86 M_{\odot}$, respectively. Taking the values of $M \sin ^{3} i$ obtained from our orbital solution into account, we estimate the inclination of the system to be about $70^{\circ}$. For BD+15 4538 we deduce masses of about 0.92 and $0.74 M_{\odot}$ for the primary and secondary components, respectively. The system inclination, estimated on the basis of the very low values of $M \sin ^{3} i$, is only about $10^{\circ}$.

The case of BD+38 2140 is a little puzzling because we obtained a spectral type $\mathrm{K} 1 \mathrm{IV}$ and a minimum radius $R \sin i \simeq$ $3.5 R_{\odot}$, but its position on the HR diagram is consistent with a main-sequence star. In our opinion, the value of the TYCHO parallax for this object could be wrong or its error could have been underestimated.

\subsection{Chromospheric and coronal activity}

Since the beginning of X-ray astronomy, different authors have demonstrated the good correlation of chromospheric and coronal activity (e.g. Schrijver 1983; Schmitt et al. 1985; Maggio et al. 1987). In the following subsections we investigate the chromospheric and coronal activity of our binaries by means of $\mathrm{H} \alpha$ and X-ray emissions, respectively.

\subsubsection{Chromospheric activity}

The $\mathrm{H} \alpha$ line is one of the most important and easily accessible indicators of chromospheric activity. Only the very active stars show the $\mathrm{H} \alpha$ line as an emission feature that is always above the local continuum, while only a filled-in absorption line is observed in the spectra of less active objects. The detection of the chromospheric emission contribution filling in the line core is hampered by the complexity of the observed spectra of double-lined binaries in which the spectral lines of both components, shifted at different wavelengths according to the orbital phase, are simultaneously seen. Therefore a comparison with a "non-active" template, composed of two stellar spectra mimicking the two components in the absence of activity, is needed to emphasize the $\mathrm{H} \alpha$ chromospheric emission. The non-active templates were built up with rotationally broadened ELODIE archive spectra or with OAC spectra acquired during the observing campaigns.

We used ELODIE spectra of HD 165341 (K0 V) and HD 10476 (K1 V) for mimicking the primary and secondary component of HD 183957, respectively. The relative contribution of the two stars in the $\mathrm{H} \alpha$ region, $w_{\mathrm{P}, \mathrm{S}}=0.56,0.44$, was found with COMPO2, the code for the synthesis of binary spectra. The two components of HD 183957 show only a small filling in their $\mathrm{H} \alpha$ profiles (Fig. 5), i.e. a moderate activity level. This agrees with the low $v \sin i$ values indicating a system composed of two early-K stars that are probably synchronously rotating with a period of about 8 days. The filling in of the $\mathrm{H} \alpha$ line of both components of HD 183957 is lower than that found for HD 166 (Biazzo et al. 2005), a K0 V star belonging to the Local Association (100-300 Myr) with a rotation period of 6.2 days, which is consistent with the decay of magnetic activity with the decrease of the rotation rate. 

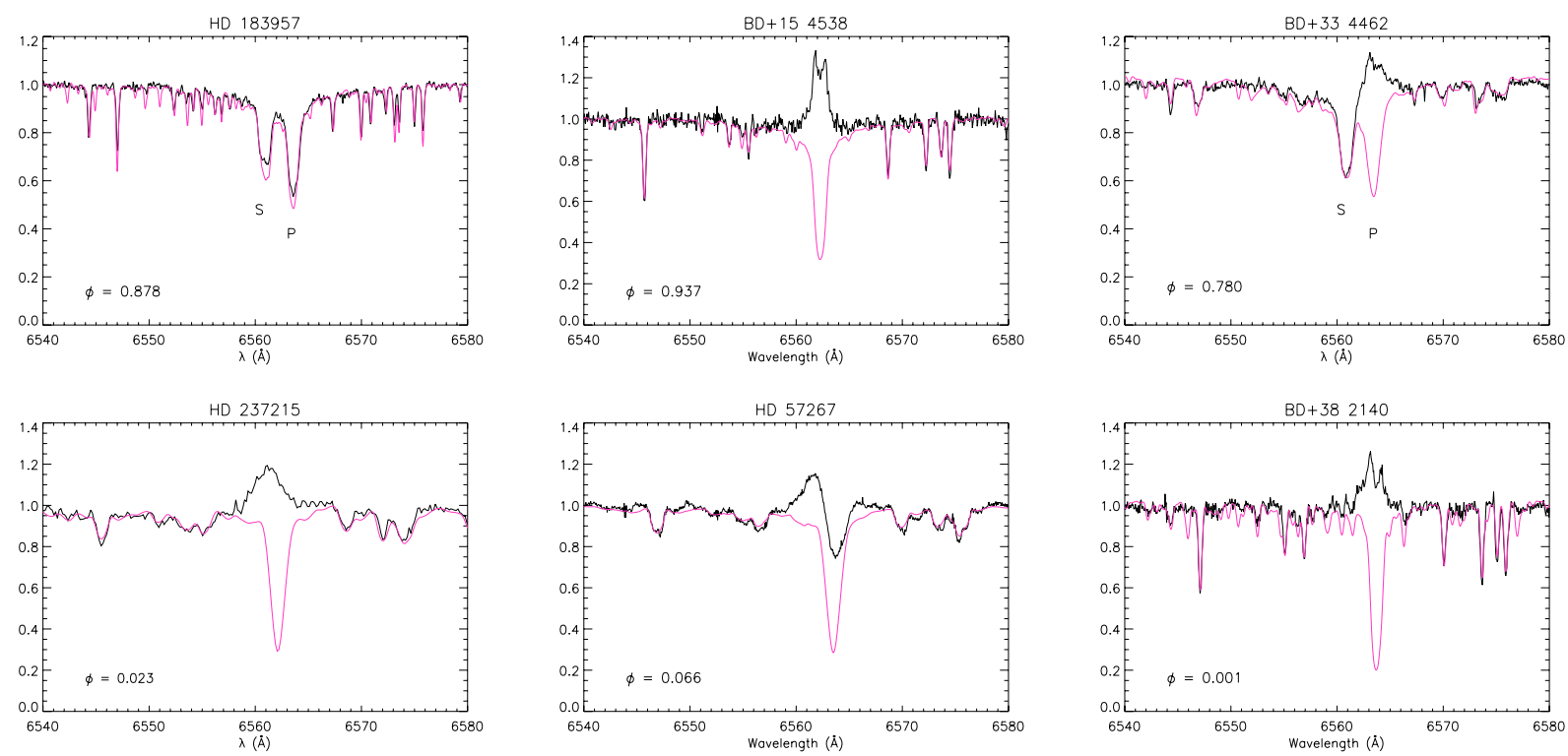

Fig. 5. Sample of spectra of the RasTyc binaries in the $\mathrm{H} \alpha$ region (thick lines). The non-active templates are displayed with thin lines. Notice the different $\mathrm{H} \alpha$ activity levels and profile shapes.

The synthetic spectrum for $\mathrm{BD}+154538$, the other binary composed of two low-mass main-sequence stars, was built up with ELODIE spectra of $\sigma$ Dra $(\mathrm{K} 0 \mathrm{~V})$ for the primary component and HD 32147 (K3 V) for the secondary one with relative contributions at $6500 \AA$ of $w_{\mathrm{P}, \mathrm{S}}=0.86,0.14$. The observed spectrum of $\mathrm{BD}+154538$ displays a strong $\mathrm{H} \alpha$ emission line with central reversal that could be mainly due to the primary component, though the lines of the two stars are strongly blended at this phase.

The ELODIE spectra of BD+33 4462 always show $\mathrm{H} \alpha$ emission above the continuum, but with a variable intensity, a situation associated with the cooler and more-massive K0 III-IV component. The hotter F7 IV component displays instead a normal $\mathrm{H} \alpha$ profile (Fig. 5). This system looks very similar to HD 114519 (F5 V+K0 IV), the prototype of the RS CVn class, both for the spectral type and the observed $\mathrm{H} \alpha$ profile of the two components.

For the SB1 systems we simply used, as non-active templates, the rotationally broadened spectra of the standard stars giving the best reproduction of the target spectra with the ROTFIT code.

An OAC spectrum of $\alpha$ Ari (K2 III) broadened at $35 \mathrm{~km} \mathrm{~s}^{-1}$ was used for HD 237215. The H $\alpha$ line of HD 237215 has been always observed as an emission feature in all OAC and AURELIE spectra, though with a variable intensity. This is behavior typical of the most active RS CVn stars like HR 1099 and DM UMa. The $\mathrm{H} \alpha$ emission peak of the spectrum displayed in Fig. 5 appears blue-shifted at that phase of the observation. According to its $\mathrm{H} \alpha$ behavior and spectral classification, HD 237215 is then very likely to be an active binary, despite its classification as a semi-regular variable star reported in Simbad and due to the behavior of the photometric variability in HIPPARCOS data.

The non-active template for HD 57267 was an ELODIE spectrum of $\delta$ Boo (G8 III) broadened at $v \sin i=40 \mathrm{~km} \mathrm{~s}^{-1}$. For this single-lined binary we observed a very broad, complex $\mathrm{H} \alpha$ profile with a filled-in core and an emission blue wing, similar to those observed in some long-period RS CVn binaries, like HK Lac (e.g. Catalano \& Frasca 1994; Biazzo et al. 2006). Similar to HD 237215, HD 57267, classified as a semi-regular variable after HIPPARCOS, also displays all the characteristics of an RS CVn SB1 binary.

Finally, an asymmetric $\mathrm{H} \alpha$ emission profile with central reversal was observed for $\mathrm{BD}+382140$. The $\mathrm{H} \alpha$ emission appears slightly blue-shifted with respect to the line center of the $\mathrm{H} \alpha$ absorption of the non-active template ( $\gamma$ Cep, K1 IV) aligned with the photospheric absorption lines of the RasTyc source (Fig. 5).

The net $\mathrm{H} \alpha$ equivalent width, $W_{\mathrm{H} \alpha}^{\mathrm{em}}$, was measured (Table 10) in the residual spectra (observed - template) by integrating the net emission profile (see, e.g., Frasca \& Catalano 1994; Frasca et al. 2002). For SB2 systems, we corrected the values of $W_{\mathrm{H} \alpha}^{\mathrm{em}}$ of each component by dividing them for the proper fractional flux contribution.

The $\mathrm{H} \alpha$ luminosity, which is a parameter more indicative of the chromospheric activity level, was calculated from the net $\mathrm{H} \alpha$ equivalent width, the distance $d$, and the continuum Earth flux at the $\mathrm{H} \alpha$ wavelength, $f_{6563}$, according to the following equation:

$$
\begin{aligned}
L_{\mathrm{H} \alpha} & =4 \pi d^{2} f_{6563} W_{\mathrm{H} \alpha}^{\mathrm{em}} \\
& =4 \pi d^{2} \frac{F_{6563}}{F_{5556}} 10^{\left(-0.4 V_{0}-8.451\right)} W_{\mathrm{H} \alpha}^{\mathrm{em}},
\end{aligned}
$$

where $10^{\left(-0.4 V_{0}-8.451\right)}$ is the Earth flux at $5556 \AA$ of a $V_{0}$-magnitude star (Gray 1992) and the continuum flux-ratio $\frac{F_{6563}}{F_{5556}}$ has been evaluated thanks to NextGen synthetic lowresolution spectra (Hauschildt et al. 1999).

\subsubsection{Coronal activity}

Computing X-ray luminosity $\left(L_{X}\right)$ from the ROSAT PSPC count-rate requires the knowledge of the source distance and of the energy conversion factor (ECF). The latter depends on the characteristics of the X-ray instrumentation, on the assumed emitted spectrum of the source, and on the amount of absorbing material on the line of sight.

On the basis of Einstein data, Pallavicini et al. (1981) and Majer et al. (1986) have established the high X-ray luminosity level and high coronal temperature of RS CVn systems. Spectral fitting of IPC photons required a model with two emitting regions at different temperatures, the lower at 2-3 million 
Table 10. $\mathrm{H} \alpha$ and X-ray luminosity.

\begin{tabular}{lccc}
\hline \hline Name & $\begin{array}{c}W_{\mathrm{H} \alpha}^{\mathrm{em}} \\
(\AA)\end{array}$ & $\begin{array}{c}\log L_{\mathrm{H} \alpha} \\
\left(\mathrm{erg} \mathrm{s}^{-1}\right)\end{array}$ & $\begin{array}{c}\log L_{\mathrm{X}} \\
\left(\mathrm{erg} \mathrm{s}^{-1}\right)\end{array}$ \\
\hline HD 183957 & $0.06 / 0.13$ & $28.06 / 28.26$ & 28.71 \\
BD+154538 & $1.99 /-$ & 29.51 & 30.21 \\
BD+334462 & $1.94 /-$ & - & - \\
HD 237215 & 2.01 & 31.87 & 31.84 \\
HD 57267 & 1.76 & 31.61 & 31.23 \\
BD+382140 & 1.52 & 28.96 & 29.38 \\
\hline
\end{tabular}

Kelvin (MK) and the hotter being above $10 \mathrm{MK}$. These results were confirmed by Dempsey et al. (1993), who found a similar behavior for BY Dra systems on the basis of ROSAT data. High coronal temperature in active binaries has been recently confirmed using the high-resolution spectrograph on board XMM (see for example Audard et al. 2002). Although multiple temperature material is required to account for the complex X-ray spectra of active binaries, we assumed a $0.9 \mathrm{keV}$ Raymond-Smith plasma in the following for the X-ray sources.

Regarding the absorbing material, we computed the column density assuming a mean extinction of $1.7 \mathrm{mag} / \mathrm{kpc}$ on the galactic plane $\left(|b|<5^{\circ}\right)$ and $0.7 \mathrm{mag} / \mathrm{kpc}$ out of the plane. The X-ray luminosities computed that way are listed in the last column of Table 10. Interestingly, the two binaries containing a giant component (namely HD 237215 and HD 57267) show coronal activity at the upper end of the RS CVn X-ray luminosity distribution. These high values of $L_{\mathrm{X}}$, in excess of $10^{31} \mathrm{erg} \mathrm{s}^{-1}$, are additional clues to their RS CVn nature. On the other hand, HD 183957 shows only moderate coronal activity, in agreement with the small filling of the $\mathrm{H} \alpha$ profiles of both components and the low values of $L_{\mathrm{H} \alpha}$. Although the 7.954 period does not contrast with a synchronous rotation, both its low activity level (compatible with the Hyades-like field population predicted by X-ray population model, Favata et al. 1992; Guillout et al. 1996) and the lack of photometric modulation correlated with orbital period can cast some doubt on the synchronization of the HD 183957 system. With X-ray luminosities in the range $10^{29.0}$ to $10^{30.5} \mathrm{erg} \mathrm{s}^{-1}$, compatible with Pleiades-like field population, BD+15 4538 and BD+38 2140 display all the characteristics of a new BY Dra system. We finally note that our $\mathrm{H} \alpha$ and X-ray diagnostics fully support the chromospheric/coronal activity connection.

\subsection{Lithium content and age}

The equivalent width of the lithium $\lambda 6708$ line, $W_{\mathrm{Li}}$, was measured on the ELODIE spectra of the RasTyc stars. For the three sources for which we were able to detect and measure $W_{\mathrm{Li}}$ (Table 9), namely BD+15 4538, HD 57267 and BD+382140, we used the Pavlenko \& Magazzù (1996) NLTE calculations to deduce a lithium abundance, $\log N(\mathrm{Li})$ of $1.8,1.8$, and 1.3 , respectively. Although the lithium content cannot be simply converted into a star age, the lithium abundances of these stars are lower than those of stars of the same temperature in the Pleiades cluster $(\approx 100 \mathrm{Myr})$ but are also significantly higher than those of Hyades $(\approx 600$ Myr) stars (see, e.g., Soderblom et al. 1993; Jeffries 1995, 2000). Such values of lithium abundance indicate ages a few hundred Myr, so that the X-ray emission and the fairly strong $\mathrm{H} \alpha$ chromospheric activity detected in these systems should essentially be the effect of the fast stellar rotation due to the spin/orbit synchronization rather than to a very young age.
Table 11. Kinematical parameters of the new binaries.

\begin{tabular}{lrrr}
\hline \hline Name & $\begin{array}{r}U \\
\left(\mathrm{~km} \mathrm{~s}^{-1}\right)\end{array}$ & $\begin{array}{c}V \\
\left(\mathrm{~km} \mathrm{~s}^{-1}\right)\end{array}$ & $\begin{array}{c}W \\
\left(\mathrm{~km} \mathrm{~s}^{-1}\right)\end{array}$ \\
\hline HD 183957 & $22.4 \pm 2.3$ & $-19.9 \pm 1.8$ & $-13.1 \pm 4.6$ \\
BD+154538 & $-25.3 \pm 2.6$ & $-30.8 \pm 1.4$ & $4.9 \pm 2.3$ \\
BD+334462 & - & - & - \\
HD 237215 & $-24.8 \pm 8.9$ & $6.4 \pm 15.1$ & $-34.8 \pm 15.9$ \\
HD 57267 & $34.6 \pm 0.7$ & $-17.5 \pm 3.4$ & $4.2 \pm 2.0$ \\
BD+382140 & $38.5 \pm 8.5$ & $-1.5 \pm 3.3$ & $31.8 \pm 5.4$ \\
\hline
\end{tabular}

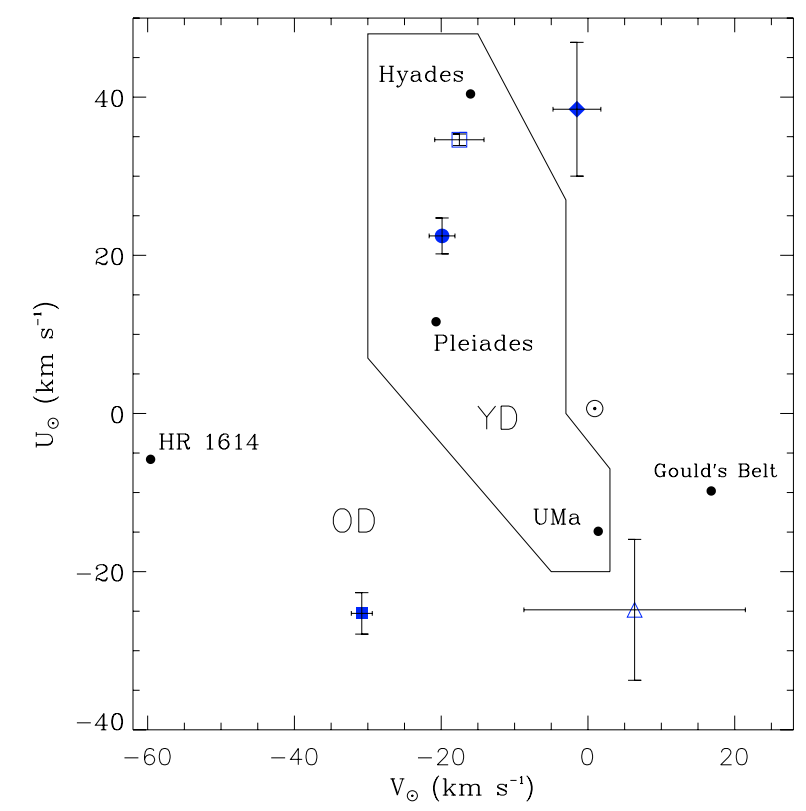

Fig. 6. Space velocities, $U, V$, of the RasTyc sources in the galactic plane. The meaning of the symbols is the same as in Fig. 4. The locus of the young-disk (YD) and the old-disk (OD) populations and the average velocity components of some moving groups (Eggen 1996) and of the Gould's Belt (Lindblad et al. 1997) are also indicated.

\subsection{Kinematics}

We derived the space-velocity components of the RasTyc binaries from their parallaxes, proper motions, and barycentric radial velocities using the formulas presented by Johnson \& Soderblom (1987). We preferred to use the heliocentric velocities in a lefthanded coordinate system, where the velocity components $U, V$, and $W$ are directed towards the galactic anti-center, the galactic rotation direction and the north galactic pole, respectively. The values of $U, V$, and $W$ are listed in Table 11 together with their errors, evaluated according to Johnson \& Soderblom (1987).

The $U-V$ diagram of the RasTyc sources is shown in Fig. 6 where the locus of the young-disk stars $\left(\right.$ age $\left.<2 \times 10^{9} \mathrm{yr}\right)$ in the solar neighborhood according to Eggen (1996) is also marked. The same diagram also displays the position of three young moving groups, namely the Pleiades supercluster $\left(\sim 10^{8} \mathrm{yr}\right)$, UMa group $\left(4-6 \times 10^{8} \mathrm{yr}\right)$ and Hyades supercluster $\left(\sim 10^{9} \mathrm{yr}\right)$. The solar-age HR 1614 supercluster $\left(\sim 4 \times 10^{9} \mathrm{yr}\right)$ and the average Gould's Belt velocity components (Lindblad et al. 1997) are also displayed in the same figure.

The space velocity of HD 183957 and HD 57267 is consistent with the young-disk population, while the very active system BD+154538 seems to be more compatible with the old-disk population. The remaining two systems (HD 237215 and BD+382140) are only marginally consistent with the young-disk population, also taking into account the errors. 
Nothing can be said about BD+33 4462 for which the parallax is not known.

\section{Conclusions}

We have discovered and fully characterized six new late-type spectroscopic binaries by means of follow-up observations of the optical counterparts of stellar X-ray sources. These sources were selected from the RasTyc sample, obtained from the crosscorrelation of the TYCHO and RASS catalogs.

An intensive spectroscopic and photometric monitoring has allowed us to obtain good radial velocity curves that provide us with the orbital parameters and light curves displaying, in nearly all cases, rotational modulation with the orbital period that suggests synchronization of the orbital and rotational motions.

We performed an accurate spectral classification of the components of the SB1 and SB2 systems by means of codes developed by us. We found two systems composed of main-sequence stars and the other ones containing at least one evolved (giant or subgiant) star. The position of these stars in the HR diagram, deduced from their HIPPARCOS or TYCHO parallaxes, confirms these results with only one exception.

The relatively low lithium content $(\log N(\mathrm{Li}) \leq 1.8)$, together with the very low (or zero) eccentricity and the synchronization of orbit and rotation, indicates that none of these systems contains PMS or very young stars. The kinematics of two systems is compatible with the locus of young-disk population stars (age $<2 \times 10^{9} \mathrm{yr}$, Eggen 1996), while the remaining systems are located in the region of old-disk stars.

The main conclusion that emerges from our analysis is that the X-ray and $\mathrm{H} \alpha$ activity displayed by these systems is mainly due to the proximity of the components of these close binary systems (that also induces the coupling of spin and orbital motions by tidal actions), rather than to a very young age.

Acknowledgements. The authors are grateful to the anonymous referee for useful suggestions. This work was supported by the Italian Ministero dell'Istruzione, Università e Ricerca (MIUR), which is gratefully acknowledged. We are grateful to the members of the staff of the OHP and OAC observatories for their support and help with the observations. This research made use of SIMBAD and VIZIER databases, operated at the CDS, Strasbourg, France.

\section{References}

Audard, M., Güdel, M., Sres, A., et al. 2002, in Stellar Coronae in the Chandra and XMM-NEWTON Era, ed. F. Favata, \& J. J. Drake, ASP Conf. Ser., 277, 65

Baranne, A., Queloz, D., Mayor, M., et al. 1996, A\&AS, 119, 373

Bevington, P. R. 1969, Data Reduction and Error Analysis for the Physical Sciences (McGraw-Hill Book Company), 237
Biazzo, K., Frasca, A., Henry, G. W., Catalano, S., \& Marilli, E. 2005, in 13th Cambridge Workshop on Cool Stars, Stellar Systems, and the Sun (2005 July 5-9), ed. F. Favata, ESA SP-560, 445

Biazzo, K., Frasca, A., Catalano, S., \& Marilli, E. 2006, A\&A, 446, 1129

Brandner, W., \& Koehler, R. 1998, ApJ, 499, L79

Catalano, S., \& Frasca, A. 1994, A\&A 287, 575

Cox, A. N. 2000, Allen's Astrophysical Quantities, 4th edn. (New York: AIP Press and Springer-Verlag)

Dempsey, R. C., Linsky, J. L., Fleming, T. A., \& Schmitt, J. H. M. M. 1993, ApJS, 86, 599

Duchêne, G. 1999, A\&A, 341, 547

Eggen, O. J. 1996, AJ, 112, 1595

ESA 1997, The Hipparcos and Tycho Catalogues, ESA SP-1200

Favata, F., Micela, G., Sciortino, S., \& Vaiana, G. S. 1992, A\&A, 256, 86

Fitzpatrick, M. J. 1993, in Astronomical Data Analysis Software and System II, ed. R. J. Hanish, R. V. J. Brissenden, \& J. Barnes, ASP Conf. Ser., 52, 472

Flower, P. J. 1996, ApJ, 469, 355

Frasca, A., \& Catalano, S. 1994, A\&A, 284, 883

Frasca, A., Freire Ferrero, R., Marilli, E., \& Catalano, S. 2000, A\&A, 364, 179

Frasca, A., Çakırlı, Ö., Catalano, S., et al. 2002, A\&A, 388, 298

Frasca, A., Alcalá, J. M., Covino, E., et al. 2003, A\&A, 405, 149

Girardi, L., Bressan, A., Bertelli, G., \& Chiosi, C. 2000, A\&AS, 141, 371

Gray, D. 1992, The Observation and Analysis of Stellar Photospheres, second edition (Cambridge University Press), 184

Guillout, P., Haywood, M., Motch, C., \& Robin, A. C. 1996, A\&A, 316, 89

Guillout, P., Sterzik, M. F., Schmitt, J. H. M. M., Motch, C., \& Neuhäuser, R. 1998, A\&A, 337, 113

Guillout, P., Schmitt, J. H. M. M., Egret, D., et al. 1999, A\&A, 351, 1003

Hauschildt, P. H., Allard, F., \& Baron, E. 1999, ApJ, 512, 377

Hog, E., Fabricius, C., Makarov, V. V., et al. 2000, A\&A, 355, L27

Jeffries, R. D. 1995, MNRAS, 273, 559

Jeffries, R. D. 2000, in Stellar Clusters and Associations: Convection, Rotation, and Dynamos, ed. R. Pallavicini, G. Micela, \& S. Sciortino, ASP Conf. Ser., 198,245

Johnson, D. R. H., \& Soderblom, D. R. 1987, AJ, 93, 864

Katz, D., Soubiran, C., Cairel, R., Adda, M., \& Cautain, R. 1998, A\&A, 338, 151

Landolt, A. U. 1992, AJ, 104, 340

Lindblad, P. O., Palous, J., Loden, K., \& Lindegren, L. 1997, in Proceedings of the ESA Symposium 'Hipparcos - Venice “97”, ESA SP-402 (July 1997), 507

Lo Presti, C., \& Marilli, E. 1993, PHOT - Photometrical Data Reduction Package, Internal report of Catania Astrophysical Observatory N. 2/1993

Lucy, L. B., \& Sweeney, M. A. 1971, AJ, 76, 544

Maggio, A., Sciortino, S., Vaiana, G. S., et al. 1987, ApJ, 315, 687

Majer, P., Schmitt, J. H. M. M., Golub, L., Harnden, F. R., Jr, \& Rosner, R. 1986, ApJ, 300, 360

Melo, C. H. F., Covino, E., Alcalá, J. M., \& Torres, G. 2001, A\&A, 378, 898

Pallavicini, R., Golub, L., Rosner, R., et al. 1981, ApJ, 248, 279

Pavlenko, Y. V., \& Magazzù, A. 1996, A\&A, 311, 961

Prugniel, P., \& Soubiran, C. 2001, A\&A, 369, 1048

Queloz, D., Allain, S., Mermilliod, J.-C., Bouvier, J., \& Mayor, M. 1998, A\&A, 335,183

Schrijver, C. J. 1983, A\&A, 127, 289

Schmitt, J. H. M. M., Golub, L., Harnden, F. R., Jr, et al. 1985, ApJ, 290, 307

Soderblom, D. R., Jones, B. F., Balachandran, S., et al. 1993, AJ, 106, 1059

Tonry, J., \& Davis, M. 1979, AJ, 84, 1511

Topping, J. 1972, Errors of Observation and Their Treatment (Chapman and Hall Ltd.), 89

Wichmann, R., Schmitt, J. H. M. M., \& Hubrig, S. 2003, A\&A, 399, 983

Zahn, J.-P., \& Bouchet, L. 1989, A\&A, 223, 112

Zickgraf, F.-J., Krautter, J., Reffert, S., et al. 2005, A\&A, 433, 151 